\title{
Global Analysis of a Discrete Nonlocal and Nonautonomous Fragmentation Dynamics Occurring in a Moving Process
}

\author{
E. F. Doungmo Goufo and S. C. Oukouomi Noutchie \\ Department of Mathematical Sciences, North-West University, Mafikeng 2735, South Africa \\ Correspondence should be addressed to E. F. Doungmo Goufo; franckemile2006@yahoo.ca
}

Received 12 September 2013; Accepted 28 September 2013

Academic Editor: Abdon Atangana

Copyright ( 2013 E. F. Doungmo Goufo and S. C. Oukouomi Noutchie. This is an open access article distributed under the Creative Commons Attribution License, which permits unrestricted use, distribution, and reproduction in any medium, provided the original work is properly cited.

\begin{abstract}
We use a double approximation technique to show existence result for a nonlocal and nonautonomous fragmentation dynamics occurring in a moving process. We consider the case where sizes of clusters are discrete and fragmentation rate is time, position, and size dependent. Our system involving transport and nonautonomous fragmentation processes, where in addition, new particles are spatially randomly distributed according to some probabilistic law, is investigated by means of forward propagators associated with evolution semigroup theory and perturbation theory. The full generator is considered as a perturbation of the pure nonautonomous fragmentation operator. We can therefore make use of the truncation technique (McLaughlin et al., 1997), the resolvent approximation (Yosida, 1980), Duhamel formula (John, 1982), and Dyson-Phillips series (Phillips, 1953) to establish the existence of a solution for a discrete nonlocal and nonautonomous fragmentation process in a moving medium, hereby, bringing a contribution that may lead to the proof of uniqueness of strong solutions to this type of transport and nonautonomous fragmentation problem which remains unsolved.
\end{abstract}

\section{Introduction and Useful Definitions}

Fragmentation models have attracted considerable attention lately as they can be found in many important areas of science and engineering. Examples range from the splitting of phytoplankton clusters, astrophysics, rock crushing, colloidal chemistry, and polymer science to depolymerization. The dynamical behavior of a nonautonomous system of phytoplankton clusters, for example, which are undergoing breakup to produce smaller particles in a moving medium can be derived by balancing loss and gain of clusters of size $n$ (with position $x$ ) over a short period of time and is given by the following differential equation as presented in [1]:

$$
\begin{aligned}
\frac{\partial}{\partial t} p(t, x, n)= & -\operatorname{div}(\omega(x, n) p(t, x, n)) \\
& +\sum_{m=n+1}^{\infty} a(t, x, m) b(t, x, n, m) p(t, x, m) \\
& -a(t, x, n) p(t, x, n),
\end{aligned}
$$

$$
\begin{gathered}
p\left(\tau_{0}, x, n\right)=p_{\tau_{0}}(x, n), \\
0 \leq \tau_{0}<t \leq T, \quad n=1,2,3, \ldots, x \in \mathbb{R}^{3},
\end{gathered}
$$

where $p(t, x, n)$ is the particle mass distribution function with respect to the mass $n$ at the position $x \in \mathbb{R}^{3}$ and time $t,\left(p_{\tau_{0}}(x, n) \equiv p_{n, \tau_{0}}(x)\right.$ is the mass distribution at some fixed time $\left.\tau_{0} \geq 0\right), b(t, x, n, m) \equiv b_{n, m}(t, x)$ is the distribution of particle masses $n$ and position $x$, spawned by the fragmentation of a particle of mass $m$ at time $t, T \in \mathbb{R}$, and $a(t, x, n) \equiv a_{n}(t, x)$ is the evolutionary time-dependent fragmentation rate, that is, the rate at which mass $n$ particles at position $x$ break up at a time $t$. The velocity $\omega=\omega(x, n)$ of the transport is supposed to be a known quantity, depending on the size $n$ of aggregates and their position $x$.

The combination of fragmentation equations and transport mechanisms have been successfully utilized to model a wide range of natural processes. Examples in chemical engineering include polymer breakup and solid drugs degradation in fluids. In aquaculture, such models are used to 
describe phytoplankton dynamics under the kinetic constraints of the flow. The mathematical investigation of fragmentation models presents several challenges both from the theoretical and numerical point of view. In [2] the authors investigated the existence of global solutions to continuous nonlocal convection-fragmentation equations in spaces of distributions with finite higher moments. But till now, models with time dependent coefficients (nonautonomous) remain barely touched. Moreover, models of transport and nonautonomous fragmentation process have not yet been studied in the same work and there are still only few papers devoted to their analysis (well-posedness, conservativeness, honesty,...) separately or in the same model. In [3], the authors used techniques of truncation to prove existence, uniqueness, and mass conservation for a pure nonautonomous fragmentation model under certain conditions on initial data and the associated truncated system. The authors in [4] used evolution semigroups approach which allows them to build on the substochastic semigroup theory and obtain an equivalent result as in [3]. In the analysis of the book by Kato [5] and later improved by Da Prato and Grisvard [6], it is generally assumed that the generators $A(t)$ and $B(t)$ involved in the perturbation are of class $\mathscr{G}(1,0)$. This condition is modified in [7] where the authors used semigroup perturbation and renormalization approach to show that the closure of the involved operators is an antigenerator. However, in many applications of forward propagator (evolution semigroup) theory to evolution equations like transport equations used in this paper or population equations $[8,9]$, perturbation method remains essential no matter which generator is the perturbed or the perturbing operator.

As in [10], we focus on the case where after clusters fragmentation, new originating groups have different centers distributed according to a given probabilistic law $h(\cdot, n, m, y)$. This is the probability density that after a break up of an $m$ aggregate (with the center at $y$ ), the new formed $n$-group will be located at $x$. Therefore

$$
\int_{\mathbb{R}^{3}} h(x, n, m, y) d x=1,
$$

and the system (1) becomes

$$
\begin{aligned}
\frac{\partial}{\partial t} p(t, x, n)= & -\operatorname{div}(\omega(x, n) p(t, x, n)) \\
& +\sum_{m=n+1}^{\infty} \int_{\mathbb{R}^{3}} a(t, y, m) b(t, y, n, m) \\
& \times h(x, n, m, y) p(t, y, m) d y \\
& -a(t, x, n) p(t, x, n), \\
& p\left(\tau_{0}, x, n\right)=p_{\tau_{0}}(x, n), \\
0 \leq \tau_{0}< & t \leq T, \quad n=1,2,3, \ldots, x \in \mathbb{R}^{3} .
\end{aligned}
$$

Since a group of size $m \leq n$ cannot split to form a group of size $n$, we require

$$
b_{n, m}(t, x)=0
$$

at any time $t$ and position $x$ for all $m \leq n$. We also set

$$
a_{1}(t, x)=0, \quad \sum_{m=1}^{n-1} m b_{m, n}(t, x)=n \quad(n=2,3, \ldots),
$$

meaning that a cluster of size one cannot split and the sum of all individuals obtained by fragmentation at a position $x$ of an $n$-group is equal to $n$ all the time $t$. The second term on the right-hand side of (3) describes the increase in the number of particles of size $n$ due to fission of larger particles (the gain due to the fragmentation). The third term describes the reduction in the number of particles of size $n$ due to the fission of groups of the same size (the loss due to the fragmentation). The space variable $x$ is supposed to vary in the whole of $\mathbb{R}^{3}$.

\section{Approximation and Analysis of the Fragmentation Operator}

Since $p=p(t, x, n)$ is the density of $n$-groups at the position $x$ and time $t$ and that total mass

$$
U(t)=\sum_{n=1}^{\infty} n \int_{\mathbb{R}^{3}} p(t, x, n) d x
$$

is expected to be a conserved quantity, the most appropriate Banach space to work in is the space

$$
\mathscr{X}_{1}:=L_{1}\left(\mathbb{R}^{3} \times \mathbb{N}, d \mu d m_{1}\right),
$$

where $\mathbb{N}$ is equipped with the weighed counting measure $d m_{1}$ with weight $n$ and $d \mu=d x$ is the Lebesgue measure in $\mathbb{R}^{3}$. Note that elements' norm of $\mathscr{X}_{1}$ represents the total mass (or total number of individuals) of the system.

Now we recast (3) as the nonautonomous abstract Cauchy problem in $\mathscr{X}_{1}$ :

$$
\begin{gathered}
\frac{\partial}{\partial t} p(t, x, n)=[\widetilde{\mathscr{D}} p](t, x, n)+Q(t) p(t, x, n), \\
p\left(\tau_{0}, x, n\right)=p_{\tau_{0}}(x, n), \\
0 \leq \tau_{0}<t \leq T, \quad n=1,2,3, \ldots, x \in \mathbb{R}^{3},
\end{gathered}
$$

or in the compact form

$$
\begin{gathered}
\frac{\partial}{\partial t} \mathbf{p}(t)=\mathbf{D} \mathbf{p}(t)+\mathscr{Q}(t) \mathbf{p}(t), \\
\left.\mathbf{p}(t)\right|_{t=\tau_{0}}=\mathbf{p}_{\tau_{0}},
\end{gathered}
$$

where $\mathbf{p}(t)$ is the vector $\mathbf{p}(t)=(p(t, x, n))_{n=1}^{\infty}, \mathbf{p}_{\tau_{0}}$ the mass distribution vector $\left(p_{n}\left(\tau_{0}, x\right)\right)_{n=1}^{\infty}$ at the fixed time $\tau_{0} \geq 0$ 
and position $x$, and $Q(t)$ the nonautonomous fragmentation operator defined by

$$
\begin{aligned}
\mathcal{Q}(t) \mathbf{p}(t)=([Q(t) p(t)](t, x, n))_{n=1}^{\infty} \\
:=\left(\sum_{m=n+1}^{\infty} \int_{\mathbb{R}^{3}} a(t, y, m) b(t, y, n, m)\right. \\
\quad \times h(x, n, m, y) p(t, y, m) d y \\
\quad-a(t, x, n) p(t, x, n))_{n=1}^{\infty},
\end{aligned}
$$

where $Q(t)$ is seen as the pointwise operation

$$
\begin{gathered}
\psi(t, x, n) \longmapsto \sum_{m=n+1}^{\infty} \int_{\mathbb{R}^{3}} a(t, y, m) b(t, y, n, m) \\
\quad \times h(x, n, m, y) \psi(t, y, n) d y \\
-a(t, x, n) \psi(t, x, n)
\end{gathered}
$$

defined on the set of measurable functions. $Q(t)$ is defined by $Q(t)=\mathfrak{Q}(t)$ and represents the realization of $\mathfrak{Q}(t)$ on the domain

$$
D(Q(t))=\left\{p \in \mathscr{X}_{1} ; \mathfrak{Q}(t) p(t) \in \mathscr{X}_{1}\right\},
$$

with $\mathfrak{Q}(t) p(t)$ given by (11). The transport matrix $\mathbf{D}$ is given by $\mathbf{D p}:=(\widetilde{\mathscr{D}} p(t, x, n))_{n=1}^{\infty}$ with

$$
[\widetilde{\mathscr{D}} p](t, x, n)=-\operatorname{div}(\omega(x, n) p(t, x, n)) .
$$

To investigate and analyze the abstract Cauchy Problem (8) and show existence for this system, we will need a two parameter family.

Definition 1 (evolution system [11] or propagator [7]). A two parameter family of bounded linear operators $U(t, \tau), 0 \leq$ $\tau \leq t \leq T$, on a Banach space is called forward propagator or evolution system if the following conditions are respected:

(i) $U(\tau, \tau)=I$ for $0 \leq \tau \leq T$;

(ii) $U(t, r) U(r, \tau)=U(t, \tau)$ for $0 \leq \tau \leq r \leq t \leq T$;

(iii) $(t, \tau) \rightarrow U(t, \tau)$ is strongly continuous for $0 \leq \tau \leq$ $t \leq T$.

Recall that [7] the forward propagator $U(t, \tau), 0 \leq \tau \leq t \leq$ $T$ can be associated with the so-called evolution semigroup $\left(G_{t}(s)\right)_{s \geq 0}$ defined in $\mathscr{X}_{1}$; that is, if for any fixed $t \in \mathfrak{J}=[0, T]$, the operator $Q(t)$ generates a forward propagator $U(t, \tau), 0 \leq$ $\tau \leq t \leq T$, then this propagator defines a $C_{0}$ semigroup $\left(G_{t}(s)\right)_{s \geq 0}$ given by the relation

$$
\left[G_{t}(s) p\right](\sigma)=(\sigma-s) \chi_{\mathfrak{J}} U(\sigma, \sigma-s) p(\sigma-s),
$$

where $\chi_{\mathfrak{J}}$ is the characteristic function of $\mathfrak{\Im}=[0, T], p \in$ $\mathscr{X}_{1}$, and $\sigma \in \mathfrak{J}$. In the following pages, when we say $Q(t)$ is the generators of $C_{0}$ semigroups in $\mathscr{X}_{1}$, we mean that $Q(t)$ generates a propagator which defines a $C_{0}$ semigroup in $\mathscr{X}_{1}$ satisfying the relation (14).
We introduce, for any given $k \in \mathbb{N}$, the projection operator $q_{k}$ defined for a function $g \in \mathscr{X}_{1}$ as

$$
\left[q_{k} g\right](x, n)= \begin{cases}g(x, n), & k>n>0, x \in \mathbb{R}^{3} \\ 0, & \text { otherwise }\end{cases}
$$

The space

$$
X_{k}=\left\{g \in X_{1}: g(x, n) \equiv 0 \text { on } \mathbb{R}^{3} \times(k, \infty)\right\}
$$

is therefore a closed subspace of $\mathscr{X}_{1}$ on which the projection operator $q_{k}$ acts. Let us associate with the fragmentation model

$$
\begin{gathered}
\frac{d p}{d t}(t)=Q(t) p(t), \\
p\left(\tau_{0}\right)=p_{\tau_{0}}, \quad 0 \leq \tau_{0}<t \leq T,
\end{gathered}
$$

the following truncated version:

$$
\begin{gathered}
\frac{d p}{d t}(t)=Q(t) q_{k} p(t), \\
p\left(\tau_{0}\right)=p_{\tau_{0}}, \quad 0 \leq \tau_{0}<t \leq T,
\end{gathered}
$$

where $Q(t)$ is represented by (11). We set $Q_{k}(t)=Q(t) q_{k}$, then $Q_{k}(t)$ can be decomposed as $Q_{k}(t)=A_{k}(t)+B_{k}(t)$ where the loss and the gain fragmentation operators $A_{k}(t)$ and $B_{k}(t)$ are given by

$$
\begin{aligned}
A_{k}(t) g(t, x, n) & =A(t) q_{k} g(t, x, n)=-a(t, x, n) g(t, x, n), \\
B_{k}(t) g(t, x, n) & =B(t) q_{k} g(t, x, n) \\
& =\sum_{m=n+1}^{k} \int_{\mathbb{R}^{3}} a(t, y, m) b(t, y, n, m) \\
& \times h(x, n, m, y) g(t, y, m) d y,
\end{aligned}
$$

where $A(t)$ and $B(t)$ are expressed as

$$
\begin{gathered}
A_{k}(t)=A(t), \\
B(t) g(t, x, n)=\sum_{m=n+1}^{\infty} \int_{\mathbb{R}^{3}} a(t, y, m) b(t, y, n, m) \\
\times h(x, n, m, y) g(t, y, m) d y .
\end{gathered}
$$

Thus, for all $g \in \mathscr{X}_{1}, k \in \mathbb{N}$ and $(t, x, n) \in \mathfrak{I} \times \mathbb{R}^{3} \times \mathbb{N}$, 


$$
\begin{aligned}
& Q_{k}(t) g(t, x, n) \\
& \quad= \begin{cases}\sum_{m=n+1}^{k} \int_{\mathbb{R}^{3}} a(t, y, m) b(t, y, n, m) h(x, n, m, y) g(t, y, m)-a(t, x, n) g(t, x, n), & k>n>0 \\
0, & \text { otherwise, }\end{cases}
\end{aligned}
$$

with

$$
D\left(Q_{k}(t)\right)=\left\{g \in \mathscr{X}_{1} ; Q_{k}(t) g \in X_{k}\right\} .
$$

We assume that for $t$ fixed in $\mathbb{R}$, there are two constants $0<\theta_{1}$ and $\theta_{2}$ such that

$$
\begin{array}{r}
\theta_{1} \alpha(n, t) \leq a_{t}(x, n)=a(t, x, n) \leq \theta_{2} \alpha(n, t) \\
\text { for almost all }(x, n) \in \mathbb{R}^{3} \times \mathbb{N},
\end{array}
$$

where $\alpha(n, t) \in L_{\infty, \text { loc }}\left(\overline{\mathbb{R}_{+}^{2}}\right)$ is a real-valued function which can depend on $n$ and $t$ but is independent of the state variable $x$. This obviously implies that for any $k \in \mathbb{N}$ there exists a positive $\Theta_{t, k}$ such that

$$
\text { ess } \sup _{\mathbb{R}^{3} \times(0, k)} a_{t}(x, n) \leq \Theta_{t, k} \text {. }
$$

Moreover the sequence $\left\{\Theta_{t, k}\right\}_{k \in \mathbb{N}}(t$ fixed in $\mathbb{R})$ is bounded in the following way:

$$
\text { ess } \sup _{0 \leq t \leq T} \Theta_{t, k} \leq \bar{\Theta}_{T, k}<\infty \text {. }
$$

Lemma 2. For $t$ fixed in $\mathfrak{\Im}$, and $k \in \mathbb{N}$, there is a positive uniformly continuous semigroup of contractions on $X_{k} \subset$ $\mathscr{X}_{1}$, say $\left(G_{t, k}(s)\right)_{s \geq 0}$ generated by operator $Q_{k}(t)$ such that $\left(G_{t, k}(s)\right)_{s \geq 0}$ is conservative on $\left(X_{k}\right)_{+}$and given by

$$
G_{t, k}(s)=I+\sum_{j=1}^{\infty}\left[\left(\frac{s^{j}(Q(t))^{j}}{j !}\right)\right] q_{k} .
$$

Moreover, for any $r \geq k, s \geq 0$,

$$
q_{k} G_{t, r}(s) q_{k}=G_{t, r}(s) .
$$

Proof. Let us fix $t$ in $\mathfrak{\Im}$. The operator $A_{k}(t)$ is bounded by (25). Changing the order of summation by the Tonelli's theorem, for every $g \in X_{k}$,

$$
\begin{aligned}
\left\|B_{k}(t) g\right\|_{1} & \int_{\mathbb{R}^{3}}\left(\sum_{n=1}^{\infty} n \sum_{m=n+1}^{k} \int_{\mathbb{R}^{3}} a(t, y, m) b(t, y, n, m)\right. \\
& \times h(x, n, m, y)|g(t, y, m)| d y) d x \\
= & \int_{\mathbb{R}^{3}}\left(\sum_{n=1}^{\infty} n \sum_{m=n+1}^{k} a(t, y, m) b(t, y, n, m)|g(t, y, m)|\right) d y \\
= & \int_{\mathbb{R}^{3}} \sum_{m=2}^{k} a(t, y, m)|g(t, y, m)|\left(\sum_{n=1}^{\infty} n b(t, y, n, m)\right) d y \\
= & \int_{\mathbb{R}^{3}} \sum_{m=2}^{k} a(t, y, m)|g(t, y, m)|\left(\sum_{n=1}^{m-1} n b(t, y, n, m)\right) d y \\
= & \int_{\mathbb{R}^{3}} \sum_{m=2}^{k} m a(t, y, m)|g(t, y, m)| d y \\
= & \int_{\mathbb{R}^{3}} \sum_{m=1}^{k} m a(t, y, m)|g(t, y, m)| d y \\
\sum_{m=1}^{\infty} m a(t, y, m)|g(t, y, m)| d y &
\end{aligned}
$$

where we have used (2) and (5), respectively. Then $B_{k}(t)$ is also bounded. Hence $Q_{k}(t)$ generates a uniformly continuous semigroup. We denote this semigroup by $\left(G_{t, k}(s)\right)_{s \geq 0}$. Clearly, $A_{k}(t)$ generates a positive semigroup of contractions and $B_{k}(t)$ is a positive operator. Moreover, the above calculations also imply that $D\left(B_{k}(t)\right) \supset D\left(A_{k}(t)\right)$ and

$$
\begin{aligned}
\sum_{n=1}^{\infty} \int_{\mathbb{R}^{3}} n\left(A_{k}(t) g(t, x, n)+B_{k}(t) g(t, x, n)\right) d x \\
=\sum_{n=1}^{k} \int_{\mathbb{R}^{3}} n\left(A_{k}(t) g(t, x, n)+B_{k}(t) g(t, x, n)\right) d x \leq 0
\end{aligned}
$$


for all $g \in\left(D\left(A_{k}(t)\right)\right)_{+}$with

$$
\begin{gathered}
D\left(A_{k}(t)\right)=\left\{g \in X_{1} ;-a_{t} g \in X_{k}\right\}, \\
D\left(B_{k}(t)\right)=\left\{g \in X_{1} ; B_{k}(t) g \in X_{k}\right\} .
\end{gathered}
$$

Thus the assumptions of Kato's theorem in $L_{1}$-space [12, Corollary 5.17] hold. We essentially note that for each fixed $t$ the operator $Q_{k}(t)$ becomes independent of time [3, Lemma 2.1] and Kato's theorem is immediately applicable. Therefore there is an extension $\breve{Q}_{k}(t)$ of $Q_{k}(t)$ which generates a substochastic semigroup. Because $a_{t}(x, n)$ is bounded in $\mathbb{R}^{3} \times$ $(0, k)$, this substochastic semigroup is conservative and it follows that $\breve{Q}_{k}(t)=\bar{Q}_{k}(t)$, where $\bar{Q}_{k}(t)$ is the closure of $Q_{k}(t)$. Since $Q_{k}(t)$ generates a uniformly (and hence strongly) continuous semigroup, $Q_{k}(t)$ is a closed operator. Therefore we have that $\breve{Q}_{k}(t)=Q_{k}(t)$; consequently, the uniformly continuous semigroup $\left(G_{t, k}(s)\right)_{s \geq 0}$ is a positive strongly continuous semigroup of contractions and furthermore $\left(G_{t, k}(s)\right)_{s \geq 0}$ is honest.

The proof of (27) is clear since we can use the usual power series definition to define $G_{t, k}(s)=\exp \left(s Q_{k}(t)\right)$. By induction, $\left(Q_{k}(t)\right)^{J}=\left(Q(t) q_{k}\right)^{J}=(Q(t))^{J} q_{k}$ for $J=1,2, \ldots$, from which the exponential formula yields (27).

To prove (28), we have that $B(t) q_{k} g=q_{k} B(t) q_{k} g$ on $\overline{B(t)}\left(0_{\mathbb{R}^{3}}, k\right) \times[0, k]$ since for $k \geq n \geq 0, B(t) q_{k} g(t, x, n)$ is given by $(21)$ and $B(t) q_{k} g(t, x, n)=0$ for $k<n$. Moreover, it is obvious that $A(t) q_{k} g=q_{k} A(t) q_{k} g$; hence we have also

$$
\begin{aligned}
(A(t)+B(t)) q_{k} & =A(t) q_{k}+B(t) q_{k} \\
& =q_{k} A(t) q_{k}+q_{k} B(t) q_{k}=A_{k}(t)+B_{k}(t) \\
& =Q_{k}(t) .
\end{aligned}
$$

Next, by $q_{k} q_{r}=q_{r} q_{k}=q_{k}$ we have

$$
q_{k} Q_{r}(t) q_{k}=q_{k} q_{r} Q(t) q_{r} q_{k}=q_{k} Q(t) q_{k}=Q_{k}(t)
$$

if we assume, by induction, that $q_{k}\left(Q_{r}(t)\right)^{j-1} q_{k}=\left(Q_{k}(t)\right)^{j-1}$, then

$$
\begin{aligned}
q_{k}\left(Q_{r}(t)\right)^{j} q_{k} & =q_{k}\left(Q_{r}(t)\right)^{j-1} Q_{r}(t) q_{k} \\
& =q_{k}\left(Q_{r}(t)\right)^{j-1} q_{r} Q(t) q_{r} q_{k} \\
& =q_{k}\left(Q_{r}(t)\right)^{j-1} q_{r} q_{k} Q(t) q_{k} \\
& =q_{k}\left(Q_{r}(t)\right)^{j-1} q_{k} Q_{k}(t) \\
& =\left(Q_{k}(t)\right)^{j} .
\end{aligned}
$$

Now using (27) and the the fact that $Q_{r}(t)$ is a bounded operator, the semigroup generated by $Q_{r}(t)$ is expressed by

$$
\begin{aligned}
q_{k} G_{t, r}(s) q_{k} & =\sum_{n=0}^{\infty} \frac{s^{j} q_{k}\left(Q_{r}(t)\right)^{j} q_{k}}{n !} \\
& =\sum_{n=0}^{\infty} \frac{s^{j}\left(Q_{k}(t)\right)^{j}}{j !} \\
& =G_{t, r}(s),
\end{aligned}
$$

which concludes the lemma.

Next we assume that $a(t, x, n)$ satisfies the Lipschitz condition

$$
|a(t, x, n)-a(\sigma, x, n)| \leq|t-\sigma| \Lambda(x, n), \quad t, \sigma \in \mathfrak{\Im},
$$

where $\Lambda(x, n) \geq 0$ together with $\Lambda(x, n) \leq \Theta_{k}$; for all $k \geq n>$ 0 we state the following lemma.

Lemma 3. The function $t \rightarrow Q_{k}(t)$ is continuous in the uniform operator topology for each $k$ fixed in $\mathbb{N}$.

Proof. Using Fubini's theorem and assumption (36) yields

$$
\left\|Q_{k}(t) g-Q_{k}(\sigma) g\right\|_{1} \leq k \Theta_{k}|t-\sigma|\|g\|_{1}, \quad \forall g \in \mathscr{X}_{1},
$$

and the result follows.

Making use of (14) and Lemma 2, there is a forward propagator, say $\left\{U_{k}(t, \tau)\right\}_{0 \leq \tau \leq t \leq T}$ defined in $X_{k} \subset \mathscr{X}_{1}$ which is associated with the evolution semigroup $\left(G_{t, k}(s)\right)_{s \geq 0}, t \in$ $\mathfrak{J}$. The propagator $\left\{U_{k}(t, \tau)\right\}_{0 \leq \tau \leq t \leq T}$ shares certain properties with the family of semigroups $\left(G_{t, k}(s)\right)_{s \geq 0}, t \in \mathfrak{I}$, as stated in the following theorem and proved in [3, Theorem 4.1].

Theorem 4 (see [11]). For each $k \in \mathbb{N}$, the forward propagator $\left\{U_{k}(t, \tau)\right\}_{0 \leq \tau \leq t \leq T}$ generated by the family of infinitesimal generators $\left\{Q_{k}(t)\right\}_{t \in \mathfrak{I}}$ have the following properties:

(1) $U_{k}(t, \tau)$ is positive;

(2) $\left\|U_{k}(t, \tau) g\right\|_{1}=\|g\|_{1}$, for all $g \in \mathscr{X}_{1}$;

(3) $\sum_{n=0}^{\infty} n\left[U_{k}(t, \tau) g\right](t, x, n)=\sum_{n=0}^{\infty} n g(t, x, n)$, for all $g \in$ $\mathscr{X}_{1}, x \in \mathbb{R}^{3}$;

(4) $(\partial / \partial t) U_{k}(t, \tau)=Q_{k}(t) U_{k}(t, \tau), 0 \leq \tau \leq t \leq T$;

(5) $(\partial / \partial \tau) U_{k}(t, \tau)=-U_{k} Q_{k}(t, \tau), 0 \leq \tau \leq t \leq T$.

Theorem 5. The truncated problem (18) has a unique, strongly continuously differentiable, positive, mass-conserving solution for all initial data $p\left(\tau_{0}\right)=p_{\tau_{0}} \in X_{k}$. The solution is given by $p(t)=U_{k}\left(t, \tau_{0}\right) p_{\tau_{0}}\left(0 \leq \tau_{0} \leq t \leq T\right)$.

Proof. This theorem is an immediate consequence of Lemmas 2 and 3 and Theorem 4 associated with [11, Theorem 5.1].

Remark 6. According to [11, Definition 2.1, page 130, Remark 3.2 , page 138], for each fixed $k>0$, the family of infinitesimal generators $\left\{Q_{k}(t)\right\}_{t \in \mathfrak{I}}$ of $C_{0}$-semigroups on $X_{k}$ is stable with 
stability constants 1 and 0 . Moreover we have from Theorem 4

$$
\left\|U_{k}(t, \tau)\right\|_{1} \leq 1, \quad \text { for } 0 \leq \tau \leq t \leq T .
$$

\section{Cauchy Problem for the Transport Model in $\mathbb{R}^{3} \times \mathbb{N}$}

We consider the Cauchy problem for the transport equation

$$
\begin{gathered}
\frac{\partial}{\partial t} p(t, x, n)=-\operatorname{div}(\omega(x, n) p(t, x, n)), \\
p(0, x, n)=\stackrel{o}{p}_{n}(x), \quad n=1,2,3, \ldots,
\end{gathered}
$$

in the space $\mathscr{X}_{1}$.

We consider the function $\omega: \mathbb{R}^{3} \times \mathbb{N} \rightarrow \mathbb{R}^{3}$ defined by $(x, n) \rightarrow \omega(x, n)$ and recall that $\widetilde{\mathscr{D}}$ in $(13)$ is the expression appearing on the right-hand side of (39). Then

$$
\begin{aligned}
{[\widetilde{D} p] } & (t, x, n) \\
& :=-\operatorname{div}(\omega(x, n) p(t, x, n)) \\
& =(\nabla \cdot \omega(x, n)) p(t, x, n)+\omega(x, n) \cdot(\nabla p(t, x, n)) .
\end{aligned}
$$

We assume that

(H1) $\omega$ is divergence-free;

(H2) $\omega$ globally Lipschitz continuous;

then $\operatorname{div} \omega(x, n):=\nabla \cdot \omega(x, n)=0$ and $(40)$ becomes

$$
[\widetilde{\mathscr{D}} p](t, x, n):=\omega(x, n) \cdot(\nabla p(t, x, n)) .
$$

We define in $\mathscr{X}_{1}$ the operators $(\mathscr{D}, D(\mathscr{D}))$ as

$$
[\mathscr{D} p](t, x, n)=[\widetilde{\mathscr{D}} p](t, x, n),
$$

with $[\widetilde{\mathscr{D}} p](t, x, n)$ represented by $(41)$

$$
D(\mathscr{D}):=\left\{p \in \mathscr{X}_{1}, \omega \cdot(\nabla p) \mathscr{X}_{1}\right\}, \quad n \in \mathbb{N} \text {. }
$$

Remark 7. With the assumptions (H1)-(H2), it is proven [2] that $\mathscr{D}$ is the generator of a strongly continuous stochastic semigroup, say $\left(G_{\mathscr{D}}(t)\right)_{t \geq 0}$ and $\left\|G_{\mathscr{D}}(t)\right\| \leq 1=1 e^{0 t}$. Then $\mathscr{D} \epsilon$ $\mathscr{G}(1,0)$ and due to Hille Yosida's characterization, $(\mathscr{D}, D(\mathscr{D}))$ is a closed and densely defined operator satisfying for the resolvent set $\rho(\mathscr{D}) \supset[0, \infty)$ and $\|\lambda R(\lambda, \mathscr{D})\|_{1} \leq K$ for some constant $K>0$ and all $\lambda>0$. Furthermore,

(i) for any $g \in \mathscr{X}_{1}$,

$$
\lim _{\lambda \rightarrow \infty} \lambda R(\lambda, \mathscr{D}) g=g
$$

(ii) $\mathscr{D} R(\lambda, \mathscr{D})$ are bounded operators and for any $g \in$ $D(\mathscr{D})$,

$$
\lim _{\lambda \rightarrow \infty} \lambda \mathscr{D} R(\lambda, \mathscr{D}) g=\mathscr{D} g
$$

\section{Perturbed Approximated Problem}

Let $\mathscr{D}_{\lambda}=\lambda \mathscr{D} R(\lambda, \mathscr{D})$; Yosida [13] was the first to use the bounded operators $\mathscr{D}_{\lambda}$ to approximate the unbounded operator $\mathscr{D}$, for which we can define semigroups via the exponential formula

$$
e^{t \mathscr{D}}=I+\frac{t \mathscr{D}}{1 !}+\frac{t^{2} \mathscr{D}^{2}}{2 !}+\cdots
$$

We exploit this idea to analyze the following approximated problem associated with (3):

$$
\begin{gathered}
\frac{\partial}{\partial t} p(t, x, n)=Q_{k}(t) p(t, x, n)+\left[\mathscr{D}_{\lambda} p\right](t, x, n), \\
p\left(\tau_{0}, x, n\right)=p_{\tau_{0}}(x, n), \\
0 \leq \tau_{0}<t \leq T, \quad n=1,2,3, \ldots, x \in \mathbb{R}^{3} .
\end{gathered}
$$

Lemma 8. Let each $k$ be fixed in $\mathbb{N}$ and any $\lambda>0$. Under the assumptions (5), (25), (26), (36), (H1) and (H2), the operator $\left(T_{k, \lambda}(t)=Q_{k}(t)+\mathscr{D}_{\lambda}, D\left(Q_{k}(t)\right)\right),(0 \leq t \leq T)$ appearing in approximated Cauchy problem (46) is a stable generator, with the stability constants 1 and $\left\|\mathscr{D}_{\lambda}\right\|_{1}$, of a forward propagator $\left\{\mathbb{U}_{k, \lambda}(t, \tau)\right\}_{0 \leq \tau \leq t \leq T}$ associated with an evolution semigroup $\left(\mathbb{G}_{t, k, \lambda}(s)\right)_{s \geq 0}$, positive, conserving the norm $\|\cdot\|_{1}$ and given by

$$
\mathbb{G}_{t, k, \lambda}(s) g=G_{t, k}(s) g+\sum_{i=1}^{\infty} G_{\lambda}^{i}(s) g,
$$

where

$$
G_{\lambda}^{i}(s) g=\int_{0}^{s} G_{t, k}(s-v) g \mathscr{D}_{\lambda} G_{\lambda}^{i-1}(v) g d v, \quad g \in X_{k}
$$

with $\left(G_{t, k}(s)\right)_{s \geq 0}$ defined in Lemma 2.

Furthermore the problem (46) has a unique, strongly continuously differentiable, positive, mass-conserving solution for all initial data $p\left(\tau_{0}\right)=p_{\tau_{0}} \in X_{k}$. The solution is given by $p(t)=$ $\mathbb{U}_{k, \lambda}\left(t, \tau_{0}\right) p_{\tau_{0}}\left(0 \leq \tau_{0} \leq t \leq T\right)$.

Proof. Let us fix $k \in \mathbb{N}$ and $\lambda>0$. The fact that $T_{k, \lambda}(t)$ is the generator of a forward propagator comes from Lemma 2 , the Bounded perturbation theorem, and the remark (14). By Remark 6 we have $\rho\left(Q_{k}(t)\right) \supset(0, \infty)$. If $\nu>\left\|\mathscr{D}_{\lambda}\right\|_{1}$, it is obvious that $v \in \rho\left(T_{k, \lambda}(t)\right)$ (bounded perturbation) and the resolvent satisfies

$$
R\left(\nu, T_{k, \lambda}(t)\right)=\sum_{i=1}^{\infty} R\left(\nu, Q_{k}(t)\right)\left[\mathscr{D}_{\lambda} R\left(\nu, Q_{k}(t)\right)\right]^{i} .
$$

Henceforth, for any finite sequence $0 \leq t_{1} \leq t_{2} \leq \cdots \leq t_{r} \leq T$, $r=1,2, \ldots$, we have

$$
\begin{aligned}
\prod_{n=1}^{r} R & \left(\nu, T_{k, \lambda}\left(t_{n}\right)\right) \\
& =\prod_{n=1}^{r}\left[\sum_{i=1}^{\infty} R\left(\nu, Q_{k}\left(t_{n}\right)\right)\left[\mathscr{D}_{\lambda} R\left(\nu, Q_{k}\left(t_{n}\right)\right)\right]^{-1}\right] .
\end{aligned}
$$


The development of the right-hand side of (50) yields a series with the general term in the form

$$
\begin{aligned}
R\left(\nu, Q_{k}\left(t_{m}\right)\right)\left[\mathscr{D}_{\lambda} R\left(\nu, Q_{k}\left(t_{m}\right)\right)\right]^{i_{m}} \\
\quad \cdots R\left(v, Q_{k}\left(t_{1}\right)\right)\left[\mathscr{D}_{\lambda} R\left(\nu, Q_{k}\left(t_{1}\right)\right)\right]^{i_{1}},
\end{aligned}
$$

where $i_{n} \geq 0$. If $\sum_{n=1}^{r} i_{n}=i$; then using the stability of the family $Q_{k}(t)_{0 \leq \tau_{0} \leq t \leq T}$, we estimate (51) by $\left\|\mathscr{D}_{\lambda}\right\|_{1} v^{-i-r}$. Therefore

$$
\begin{aligned}
& \left\|\prod_{n=1}^{r} R\left(\nu, T_{k, \lambda}\left(t_{n}\right)\right)\right\|_{1} \\
& \quad \leq v^{-r} \sum_{i=1}^{\infty}\left(\begin{array}{c}
i \\
r
\end{array}\right)\left(\left\|\mathscr{D}_{\lambda}\right\|_{1} v^{-1}\right)^{-i}=\left[\left(\nu-\left\|\mathscr{D}_{\lambda}\right\|_{1}\right)\right]^{-1},
\end{aligned}
$$

where $\left(\begin{array}{l}i \\ r\end{array}\right)$ is the number of terms in which $\sum_{n=1}^{r} i_{n}=i$ in this series. To prove (47), we use the Duhamel equation [14]

$$
\begin{array}{r}
\mathbb{G}_{t, k, \lambda}(s) g=G_{t, k}(s) g+\int_{0}^{s} G_{t, k}(s-v) g \mathscr{D}_{\lambda} \mathbb{G}_{t, k, \lambda}(v) g d v \\
g \in X_{k}
\end{array}
$$

whose iteration leads to Dyson-Phillips series, see [15], given by

$$
\mathbb{G}_{t, k, \lambda}(s)=\sum_{i=0}^{\infty} G_{\lambda}^{i}(s), \quad \text { with } G_{\lambda}^{0}(s) g=G_{t, k}(s) g
$$

and (47) follows.

The second part of the theorem follows from Theorem 5 and Remark 7. We just need to show that the model with the transport process is conservative. We have proved [2] that the semigroup generated by the operator $\mathscr{D}$ is strongly continuous and stochastic and this implies

$$
\begin{aligned}
0 & =\int_{\mathbb{R}^{3} \times \mathbb{N}} \mathscr{D} p d \mu d m_{1}, \\
& =\int_{\mathbb{R}^{3}} \sum_{n=1}^{\infty} n \mathscr{D} p(t, x, n) d x,
\end{aligned}
$$

for all $p \in D(\mathscr{D}) t \geq 0$, which leads to

$$
\begin{aligned}
\frac{d}{d t} U(t) & =\frac{d}{d t}\left(\sum_{n=1}^{\infty} n \int_{\mathbb{R}^{3}} p(t, x, n) d x\right) \\
& =\sum_{n=1}^{\infty} n \int_{\mathbb{R}^{3}} \partial_{t} p(t, x, n) d x \\
& =\sum_{n=1}^{\infty} n \int_{\mathbb{R}^{3}} \mathscr{D} p(t, x, n) d x \\
& =0,
\end{aligned}
$$

where $U(t)$ is the total mass of the system defined in (6) and therefore proving the conservativeness of the transport process.
We know, see [3, Corollary 6.4], that under the assumptions of the previous Lemma, the Cauchy problem

$$
\begin{aligned}
& \frac{\partial}{\partial t} p(t, x, n)= \sum_{m=n+1}^{\infty} \int_{\mathbb{R}^{3}} a(t, y, m) b(t, y, n, m) \\
& \times h(x, n, m, y) p(t, y, m) d y \\
&-a(t, x, n) p(t, x, n), \\
& p\left(\tau_{0}, x, n\right)=p_{\tau_{0}}(x, n), \\
& 0 \leq \tau_{0}<t \leq T, \quad n=1,2,3, \ldots, x \in \mathbb{R}^{3}
\end{aligned}
$$

has a solution $p$ defined on the product set $[0, \infty) \times \mathbb{R} \times \mathbb{N}$ whenever $p_{\tau_{0}} \in \mathscr{X}_{1}$ and given by

$$
p(t, x, n)=\left[U\left(t, \tau_{0}\right) p_{\tau_{0}}\right](x, n), \quad n \in \mathbb{N}, 0 \leq \tau_{0}<t,
$$

where

$$
U(t, \tau) g=\lim _{k \rightarrow \infty} U_{k}(t, \tau) g
$$

for all $g \in \mathscr{X}_{1}$ with $\left\{U_{k}(t, \tau)\right\}_{0 \leq \tau<t \leq T}$ defined in Theorem 4 . In the same way, if $\left(G_{t}(s)\right)_{s \geq 0}$ is the evolution semigroup associated with $U(t, \tau)$, (see (14)) then

$$
G_{t}(s) g=\lim _{k \rightarrow \infty} G_{t, k}(s) g
$$

for all $g \in \mathscr{X}_{1}$ with $\left\{G_{t, k}(s)\right\}_{s \geq 0}$ defined in Lemma 2. Because $\mathscr{D}_{\lambda}$ is bounded, it follows that the perturbed Cauchy problem

$$
\begin{aligned}
& \frac{\partial}{\partial t} p(t, x, n)= \mathscr{D}_{\lambda} p(t, x, n) \\
&+\sum_{m=n+1}^{\infty} \int_{\mathbb{R}^{3}} a(t, y, m) b(t, y, n, m) \\
& \times h(x, n, m, y) p(t, y, m) d y \\
&-a(t, x, n) p(t, x, n), \\
& p\left(\tau_{0}, x, n\right)=p_{\tau_{0}}(x, n), \\
& 0 \leq \tau_{0}<t \leq T, \quad n=1,2,3, \ldots, x \in \mathbb{R}^{3}
\end{aligned}
$$

also has a solution $p$ defined on the product set $[0, \infty) \times \mathbb{R} \times \mathbb{N}$ whenever $p_{\tau_{0}} \in \mathscr{X}_{1}$. We can state the following.

Lemma 9. Let fix $\lambda>0$ and consider the families $\left(\mathbb{G}_{t, k, \lambda}(s)\right)_{s \geq 0}$ and $\left\{\mathbb{U}_{k, \lambda}(t, \tau)\right\}_{0 \leq \tau \leq t \leq T}$ defined in Lemma 8. The family $\left(\mathbb{G}_{t, \lambda}(s)\right)_{s \geq 0}$ defined by

$$
\mathbb{G}_{t, \lambda}(s) g=\lim _{k \rightarrow \infty} \mathbb{G}_{t, k, \lambda}(s) g, \quad g \in \mathscr{X}_{1}
$$

exists for all $0 \leq t \leq T$ and $\left(\mathbb{G}_{t, \lambda}(s)\right)_{s \geq 0}$ forms a positive, $C_{0^{-}}$ semigroup on $\mathscr{X}_{1}$ conserving the norm $\|\cdot\|_{1}$.

In the same way the family $\left\{\mathbb{U}_{\lambda}(t, \tau)\right\}_{0 \leq \tau \leq t \leq T}$ defined by

$$
\mathbb{U}_{\lambda}(t, \tau) g=\lim _{k \rightarrow \infty} \mathbb{U}_{k, \lambda}(t, \tau) g, \quad g \in \mathscr{X}_{1}
$$

exists for all $0 \leq \tau<t \leq T$ and $\left\{\mathbb{U}_{\lambda}(t, \tau)\right\}_{0 \leq \tau \leq t \leq T}$ forms $a$ forward propagator on $\mathscr{X}_{1}$ conserving the norm $\|\cdot\|_{1}$. 
Proof. Let $g \in \mathscr{X}_{1}$; from (27) and (28) we have

$$
\mathbb{G}_{t, k, \lambda}(s) q_{r} g=\mathbb{G}_{t, r, \lambda}(s) g+q_{r} g-g, \quad \text { where } k \geq r .
$$

Then

$$
\begin{aligned}
\left\|\mathbb{G}_{t, k, \lambda}(s) g-\mathbb{G}_{t, r, \lambda}(s) g\right\|_{1} & \\
\leq\left\|\mathbb{G}_{t, k, \lambda}(s) g-\mathbb{G}_{t, k, \lambda}(s) q_{r} g\right\|_{1} & \\
+\left\|g-q_{r} g\right\|_{1}=2\left\|g-q_{r} g\right\|_{1} & \longrightarrow 0, \\
\text { as } k, r & \longrightarrow \infty .
\end{aligned}
$$

Hence, the family $\left(\mathbb{G}_{t, k, \lambda}(s)\right)_{k \in \mathbb{N}}$ is a Cauchy sequence in the Banach space $\mathscr{X}_{1}$ and therefore convergent and its limits uniform in $s$ exist as $\mathbb{G}_{t, \lambda}(s): \mathscr{X}_{1} \rightarrow \mathscr{X}_{1}$. Furthermore

$$
\begin{aligned}
\left\|\mathbb{G}_{t, \lambda}(s) g\right\|_{1} & =\left\|\lim _{k \rightarrow \infty} \mathbb{G}_{t, k, \lambda}(s) g\right\|_{1} \\
& =\lim _{k \rightarrow \infty}\left\|\mathbb{G}_{t, k, \lambda}(s) g\right\|_{1}=\lim _{k \rightarrow \infty}\|g\|_{1} \\
& =\|g\|_{1},
\end{aligned}
$$

where we have used the continuity of $\|\cdot\|_{1}$ on $\mathscr{X}_{1}$ and the fact that $\left\{\mathbb{G}_{t, k, \lambda}(s)\right\}$ conserves the norm $\|\cdot\|_{1}$. Thus $\left\{\mathbb{G}_{t, \lambda}(s)\right\}_{s \geq 0}$ conserves the norm $\|\cdot\|_{1}$ :

$\left(\mathbb{G}_{t, k, \lambda}(s)\right)_{s \geq 0}$ is a semigroup, in fact

(a)

$$
\begin{aligned}
\mathbb{G}_{t, \lambda}(s+v) g & =\lim _{k \rightarrow \infty} \mathbb{G}_{t, k, \lambda}(s+v) g \\
& =\lim _{k \rightarrow \infty} \mathbb{G}_{t, k, \lambda}(s) \lim _{k \rightarrow \infty} \mathbb{G}_{t, k, \lambda}(v) g .
\end{aligned}
$$

In the same way as previously, we show, using the definition of $\left(\mathbb{G}_{t, \lambda}(s)\right)_{s \geq 0}$, that

$$
\begin{array}{r}
\left\|\mathbb{G}_{t, k, \lambda}(s) \mathbb{G}_{t, k, \lambda}(v) g-\mathbb{G}_{t, \lambda}(s) \mathbb{G}_{t, \lambda}(v) g\right\|_{1} \\
\text { as } k, r \longrightarrow 0,
\end{array}
$$

Hence

$$
\begin{array}{r}
\mathbb{G}_{t, \lambda}(s) \mathbb{G}_{t, \lambda}(v) g \longleftarrow \mathbb{G}_{t, k, \lambda}(s) \mathbb{G}_{t, k, \lambda}(v) g \\
=\mathbb{G}_{t, k, \lambda}(s+v) g \longrightarrow \mathbb{G}_{t, \lambda}(s+v) g \\
\text { as } k \longrightarrow \infty .
\end{array}
$$

This convergence, being strong in $\mathscr{X}_{1}$ finally yields $\mathbb{G}_{t, \lambda}(s) \mathbb{G}_{t, \lambda}(v)=\mathbb{G}_{t, \lambda}(s+v)$ :

(b) $\mathbb{G}_{t, \lambda}(0)=I$ since

$$
\mathbb{G}_{t, \lambda}(0) g=\lim _{k \rightarrow \infty} \mathbb{G}_{t, k, \lambda}(0) g=\lim _{k \rightarrow \infty} g=g
$$

for all $g \in \mathscr{X}_{1}$.

(c) By a similar way we show that $\lim _{s \rightarrow 0^{+}} \mathbb{G}_{t, k, \lambda} g=g$ since the limits (62) exist uniformly in $s$.

(d) $\left\{\mathbb{G}_{t, \lambda}(s)\right\}$ is positive since from the definition of $\mathbb{G}_{t, \lambda}(s)$ and [16, Corollary 5.11], there exists a subsequence $\left\{\mathbb{G}_{t, k_{\delta}, \lambda}(s) g\right\}(\delta=1,2, \ldots)$ so that

$$
\begin{array}{r}
{\left[\mathbb{G}_{t, k_{\delta}, \lambda}(s) g\right](x, n) \longrightarrow\left[\mathbb{G}_{t, \lambda}(s) g\right](x, n),} \\
\text { as } \delta \longrightarrow \infty \text { for a.e. }(x . n) \in \mathbb{R}^{3} \times \mathbb{N} .
\end{array}
$$

Because $\left\{\mathbb{G}_{t, k_{\delta}, \lambda}(s) g\right\}$ is positive then if $g>0$, this pointwise limit gives

$$
\left[\mathbb{G}_{t, \lambda}(s) g\right](x, n) \geq 0 \text { for a.e. }(x . n) \in \mathbb{R}^{3} \times \mathbb{N},
$$

and the results follow. The proof of the second part of this lemma is very similar to the proof of the first part with the additional note that the limit (63) is uniform in $t$ and $\tau$, which concludes the proof of the lemma.

The above discussions allow us to state the following existence result for an approximated discrete and nonautonomous fragmentation model in a moving medium.

Theorem 10. Let $\lambda>0$. Under the assumptions of Lemma 8 , the families defined in (62) and (63) are, respectively, an evolution semigroup and a forward propagator generated by the operator $\left(T_{\lambda}(t)=Q(t)+\mathscr{D}_{\lambda}, D(Q(t))\right),(0 \leq t \leq T)$ defining the Cauchy problem (61). Furthermore the solution given, for all initial data $p\left(\tau_{0}\right)=p_{\tau_{0}} \in \mathscr{X}_{1}$, by

$$
p(t, x, n)=\left[\mathbb{U}_{\lambda}\left(t, \tau_{0}\right) p_{\tau_{0}}\right](x, n), \quad n \in \mathbb{N}, 0 \leq \tau_{0}<t,
$$

satisfies the perturbed Cauchy problem (61).

\section{Existence Results: Discussion and Concluding Remark}

Now what happens if we tend $\lambda \rightarrow \infty$ !. We know, via (44), that the perturbed Cauchy problem (61) tends to discrete and nonautonomous fragmentation model in a moving medium (3) as $\lambda \rightarrow \infty$. For this reason, the solution obtained in Theorem 10 can serve to approximate a solution for the Cauchy problem (3). The results obtained here, where we had to deal with a two parameter family of bounded linear operators, improve the preceding ones [2,3] where the two processes involved in the system, namely, transport and nonautonomous fragmentation, were treated separately. However, the problem of characterizing the full generator is still an open problem for this type of perturbed nonautonomous and transport model.

\section{References}

[1] A. Okubo and S. A. Levin, Diffusion and Ecological Problems: Modern Perspectives, Springer, New York, NY, USA, 2nd edition, 2001.

[2] E. F. Doungmo Goufo and S. C. Oukouomi Noutchie, "Global solvability of a continuous model for non-local fragmentation dynamics in a moving medium," Mathematical Problem in Engineering, vol. 2013, Article ID 320750, 8 pages, 2013.

[3] D. J. McLaughlin, W. Lamb, and A. C. McBride, "Existence results for non-autonomous multiple-fragmentation models," Mathematical Methods in the Applied Sciences, vol. 20, no. 15, pp. 1313-1323, 1997.

[4] L. Arlotti and J. Banasiak, "Nonautonomous fragmentation equation via evolution semigroups," Mathematical Methods in the Applied Sciences, vol. 33, no. 10, pp. 1201-1210, 2010. 
[5] T. Kato, Perturbation Theory for Linear Operators, Die Grundlehren der mathematischen Wissenschaften, Band 132, Springer, New York, NY, USA, 1984.

[6] G. Da Prato and P. Grisvard, "Sommes d’opérateurs linéaires et équations différentielles opérationnelles," Journal de Mathématiques Pures et Appliquées, vol. 54, no. 3, pp. 305-387, 1975.

[7] H. Neidhardt and V. A. Zagrebnov, "Linear non-autonomous Cauchy problems and evolution semigroups," in Mathematical Physics, AMS, 2007.

[8] G. Greiner and R. Nagel, "Growth of cell populations via oneparameter semigroups of positive operators," in Mathematics Applied to Science, J. A. Goldstein, S. Rosencrans, and G. Sod, Eds., pp. 79-105, Academic Press, Boston, Mass, USA, 1988.

[9] W. Arendt, A. Grabosch, G. Greiner et al., One-Parameter Semigroups of Positive Operators, vol. 1184 of Lecture Notes in Mathematics, Springer, 1986.

[10] E. F. Doungmo Goufo and S. C. Oukouomi Noutchie, "On the honesty in nonlocal and discrete fragmentation dynamics in size and random position," ISRN Mathematical Analysis, vol. 2013, Article ID 908753, 7 pages, 2013.

[11] A. Pazy, Semigroups of Linear Operators and Applications to Partial Differential Equations, vol. 44 of Applied Mathematical Sciences, Springer, New York, NY, USA, 1983.

[12] J. Banasiak and L. Arlotti, Perturbations of Positive Semigroups with Applications, Springer Monographs in Mathematics, Springer, London, UK, 2006.

[13] K. Yosida, Functional Analysis, Springer, 6th edition, 1980.

[14] F. John, Partial Differential Equations, Springer, New York, NY, USA, 4th edition, 1982.

[15] R. S. Phillips, "Perturbation theory for semi-groups of linear operators," Transactions of the American Mathematical Society, vol. 74, pp. 199-221, 1953.

[16] J. D. Pryce, Methods of Linear Functional Analysis, Hutchinson \& Co., Ltd., London, UK, 1973. 


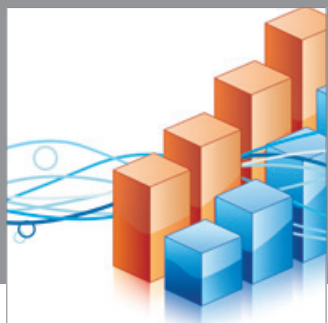

Advances in

Operations Research

mansans

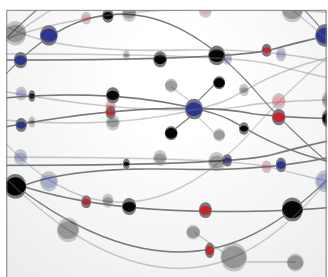

The Scientific World Journal
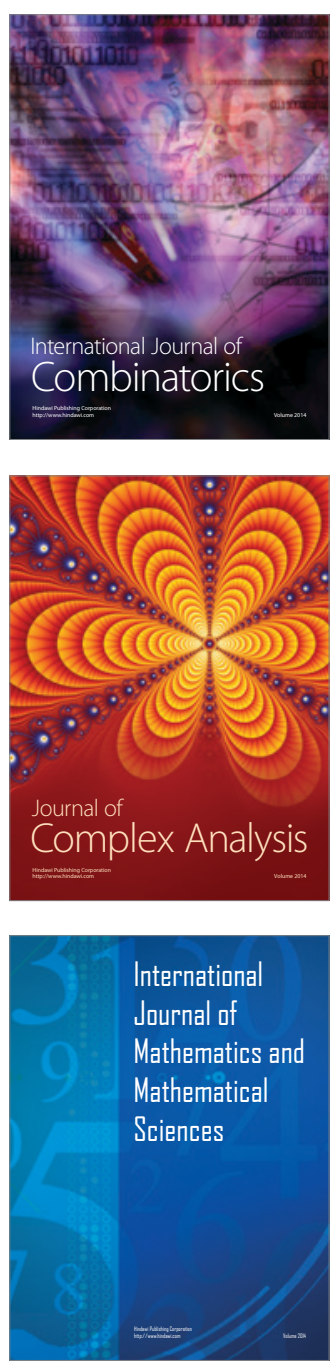
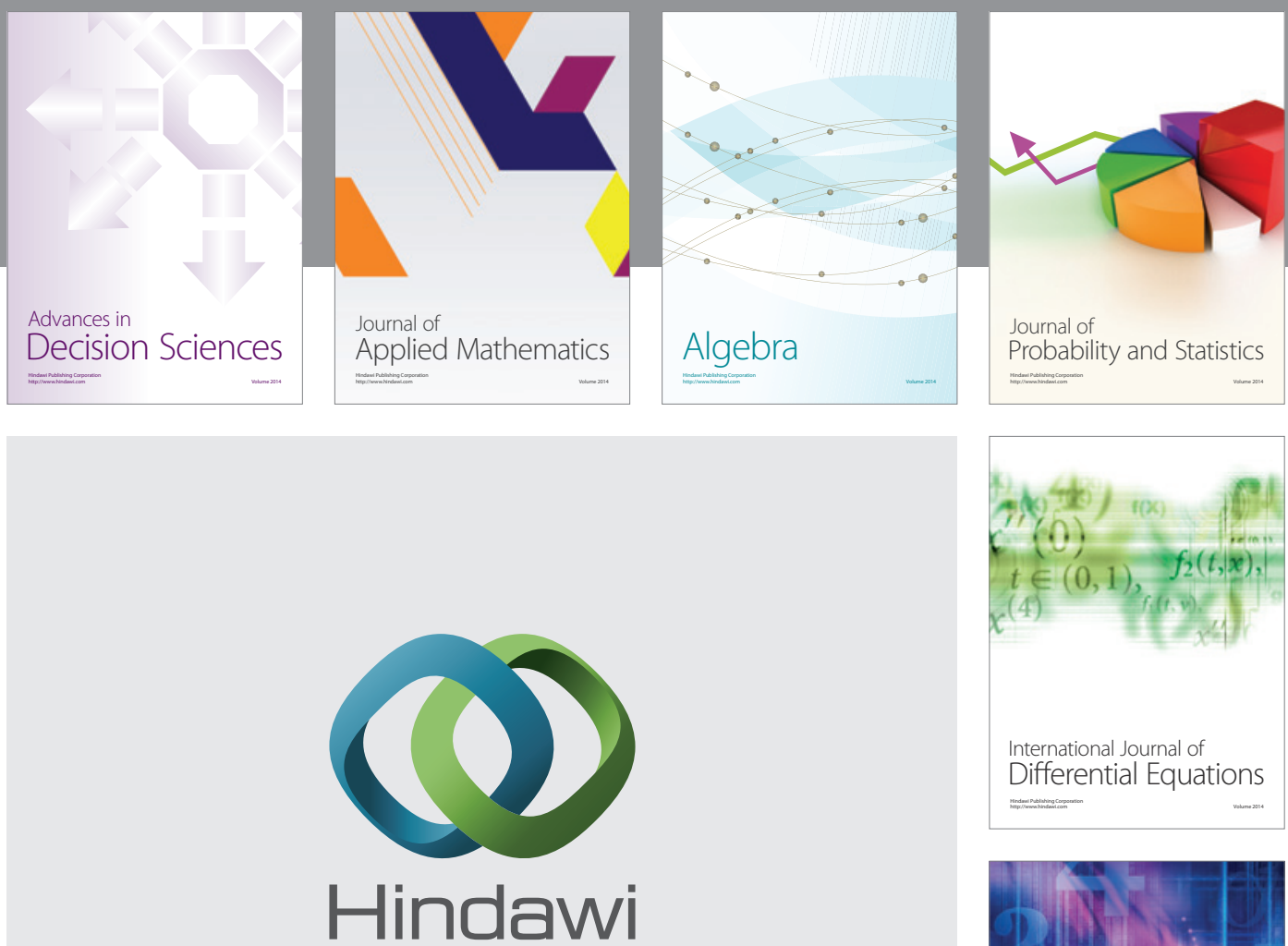

Submit your manuscripts at http://www.hindawi.com
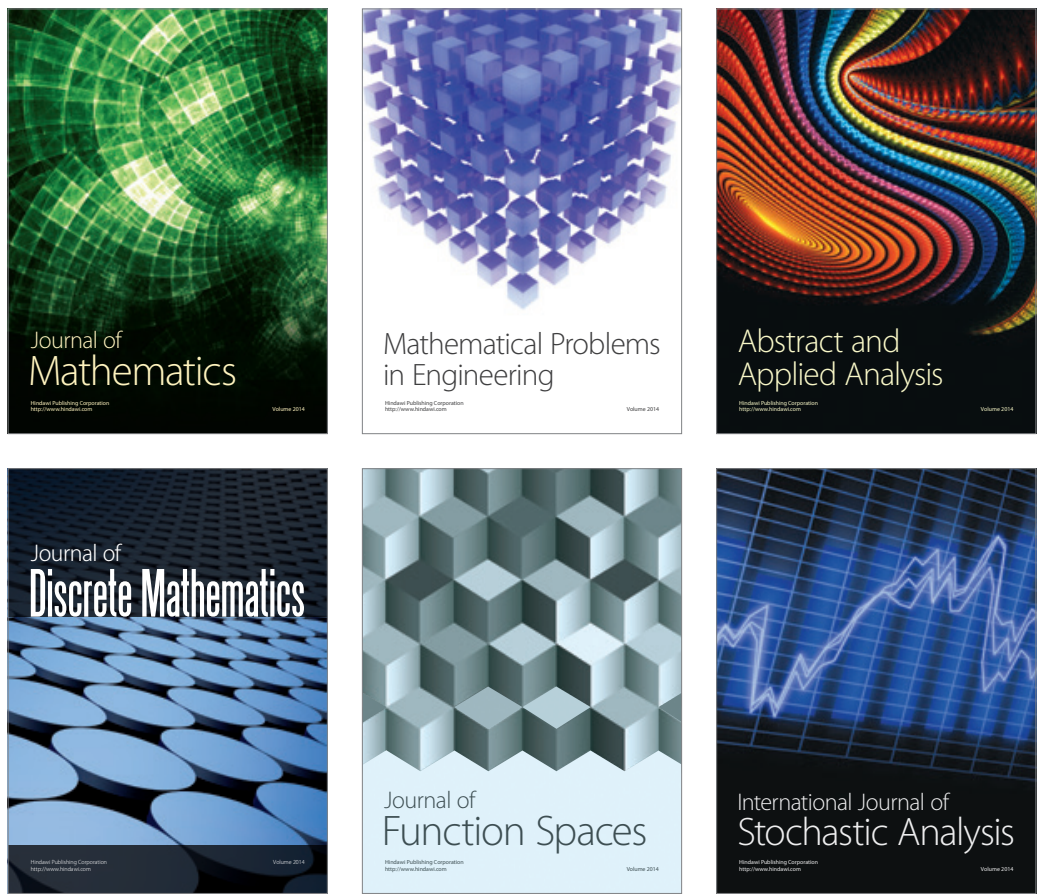

Journal of

Function Spaces

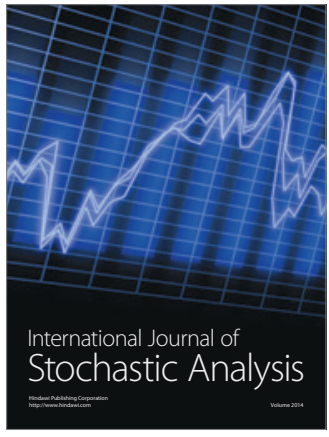

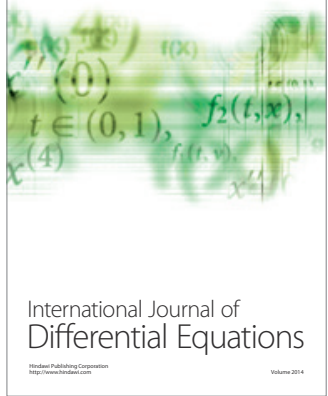
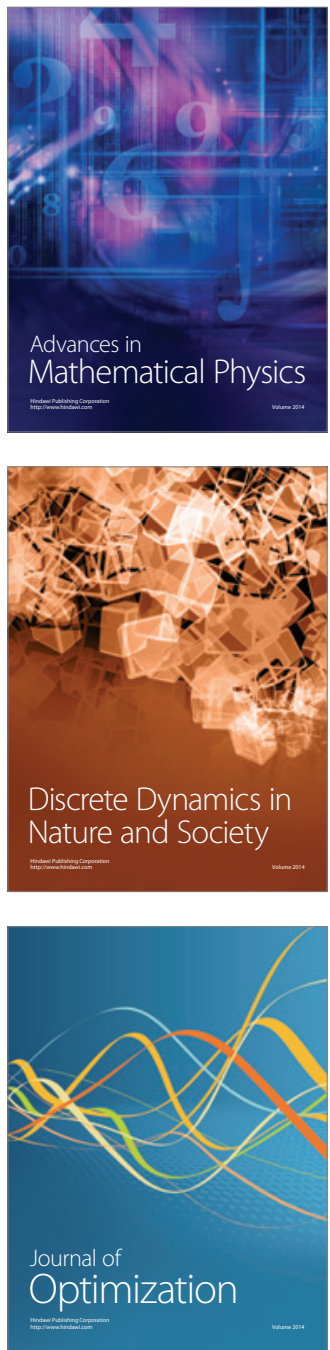